\section{Hypophosphatasia}

\author{
I J Ramage, A J Howatson, T J Beattie
}

\begin{abstract}
Hypophosphatasia is a rare inherited metabolic disease characterised by reduced plasma and tissue alkaline phosphatase activity, and may present in infancy, childhood or adulthood. The differing modes of inheritance, presentation and natural history are likely to reflect variable expression of the alkaline phosphatase gene defect. A case of infantile hypophophatasia presenting with hypercalcaemia is described and the histological and radiological resolution of the mineralisation defect present initially are reported.
\end{abstract}

(F Clin Pathol 1996;49:682-684)

Keywords: hypophosphatasia. •

Hypophosphatasia is a rare inherited metabolic disease characterised by reduced liver, bone and kidney alkaline phosphatase activity, defective bone mineralisation and increased urinary excretion of phosphoethanolamine as a result of increased bone turnover.

Variability in clinical presentation has produced subdivisions of infantile, childhood and adult forms, ${ }^{1}$ possibly arising from variability in expression of the alkaline phosphatase gene defect. Infantile hypophosphatasia may vary from a lethal perinatal form associated with virtual absence of fetal skeletal mineralisation, to severe childhood rickets.

Here, we describe a case of infantile hypophosphatasia associated with hypercalcaemia and radiological rickets and report on serial radiology and bone histology.

\section{Case report}

This infant was referred at the age of 46 days for investigation of hypercalcaemia and hypotonia. He was born at term by spontaneous vertex delivery and weighed $3.54 \mathrm{~kg}$. Within 24 hours of delivery, he developed clinical and radiological evidence of pneumonia requiring treatment with intravenous antibiotics for seven days. When 32 days old he was re-admitted with a history of pyrexia and reluctance to feed. Full blood count revealed a neutrophil leucocytosis and empirical antibiotic therapy was commenced; however, venous blood cultures demonstrated no growth and chest $x$ ray showed no abnormality.

Initial plasma investigations are shown in table 1. Urinary phosphoethanolamine was notably increased at $109 \mu \mathrm{mol} / \mathrm{mmol}$ creatinine (normal range $2-25 \mu \mathrm{mol} / \mathrm{mmol}$ ) and calcium/ creatinine ratio was $3.27 \mathrm{mmol} / \mathrm{mmol}$ (normal range $<0.7 \mathrm{mmol} / \mathrm{mmol}$ ).

Initial management involved correction of 흠 the hypercalcaemia with increased fluid intake $\frac{\mathscr{\rho}}{\widetilde{\sigma}}$ $(200 \mathrm{ml} / \mathrm{kg})$, diuretic therapy, and low calcium 0 milk formula. The plasma calcium fell tow normal within 10 days of admission.

Radiological skeletal survey carried out shortly after admission demonstrated widened $\vec{\sigma}$ cranial sutures and demineralisation of the metaphyseal regions of the long bones most: noticeable in the proximal and distal femora.. There was poor ossification of the epiphysis of the distal femora and the proximal tibia with aN normal thoracic cage. Renal ultrasound scan은 revealed evidence of nephrocalcinosis.

An initial iliac crest bone biopsy specimen taken at the age of seven months showed a들 dimorphic picture (fig 1). Most of the speci- $\stackrel{\text { ? }}{+}$ men consisted of calcified woven bone with $\overrightarrow{0}$ active osteoclastic resorption. There was also. an area formed by an anastomosing network of osteoid which was almost devoid of matrix.

Subsequent clinical and developmentalō progress was satisfactory, but the patient demonstrated early loss of primary dentition and $\stackrel{\AA}{\perp}$ required multiple extractions of carious de- $\overrightarrow{\vec{\theta}}$ ciduous teeth. Serial plasma alkaline phos- 3 phatase activities remained below 23 IU/1 but? serial plasma calcium concentrations and? urinary calcium/creatinine ratios remained normal.

Serial radiological assessment demonstrated improved skeletal mineralisation, but skull $x_{\delta}^{\delta}$ ray showed a slow progressive pansynostosis. Cranial computed tomography scan demon-윽 strated a mild degree of ventricular enlarge- $\rightarrow$ ment, and the patient currently remains under? neurosurgical review.

A further iliac crest bone biopsy was performed at the age of two years. This showed a noticeable improvement with no significant ${ }^{\omega}$ mineralisation defect (fig 2). The trabeculae were of lamellar bone and there was milde osteopenia. Active bone turnover was evidento but this was not excessive and osteoid seams were of normal thickness.

Plasma alkaline phosphatase activities were $\mathbb{\mathbb { D }}$ measured in several family members. The

Table 1 Serum biochemistry

\section{Sodium}

Potassium

Chloride

Bicarbonate

Urea

Creatinine

Calcium

Phosphate

Alkaline phophatase

1,25 Dihydroxy vitamin D

25 Hydroxy vitamin D

Correspondence to: 7 May 1996
} 
maternal activity was $42 \mathrm{IU} / 1$, and the maternal grandmother's activity was 37 IU/1 (normal adult range 30-300 IU/1). Other family members demonstrated normal plasma activities but the patient's father was not available for investigation.

\section{Discussion}

Hypercalcaemia in patients with hypophosphatasia results from reduced entry of calcium and phosphate into the skeleton. ${ }^{2}$ It is relatively common in infantile hypophosphatasia, although uncommon in the childhood and adult forms. While the diagnosis of each form of hypophosphatasia is arbitrary, the presence of hypercalcaemia, hypotonia, nephrocalcinosis, failure to thrive, and the age at presentation in this case is compatible with the diagnosis of infantile hypophosphatasia.

The genetic aspects of hypophosphatasia are incompletely understood. While it is accepted that the condition is inherited in an autosomal fashion, evidence exists for both dominant and recessive transmission, the latter being more prevalent. The differing modes of inheritance,

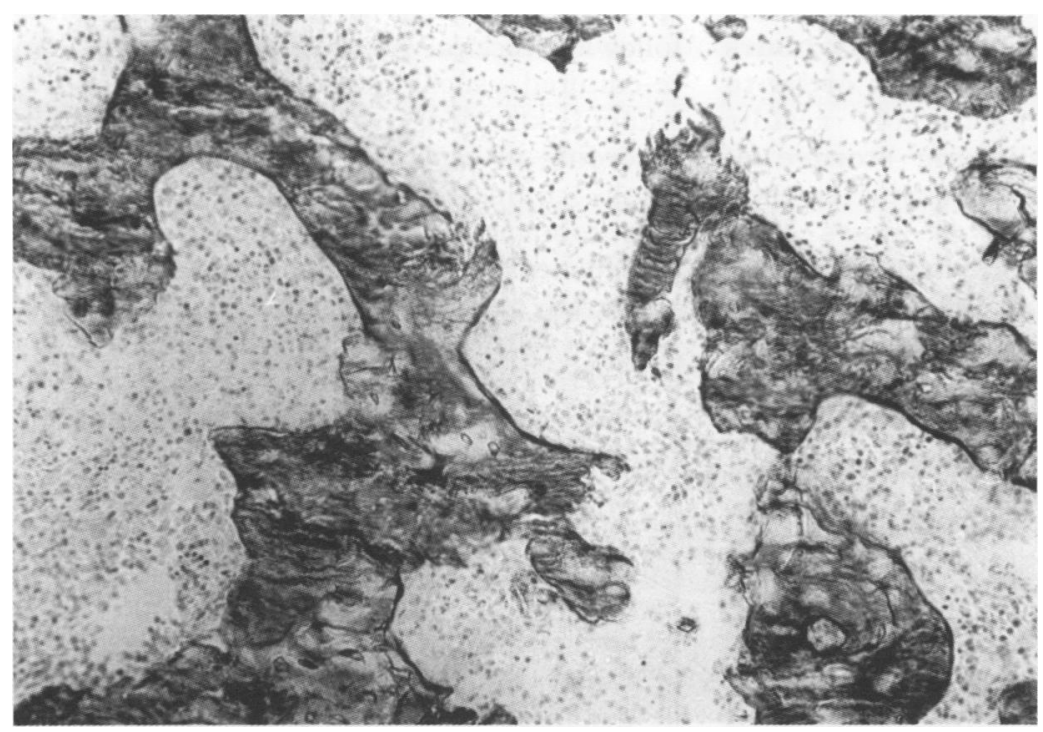

Figure 1 Right iliac crest biopsy specimen. Osteoid almost devoid of mineralised matrix. (Toluidine blue/EDTA; original magnification $\times 125$.)

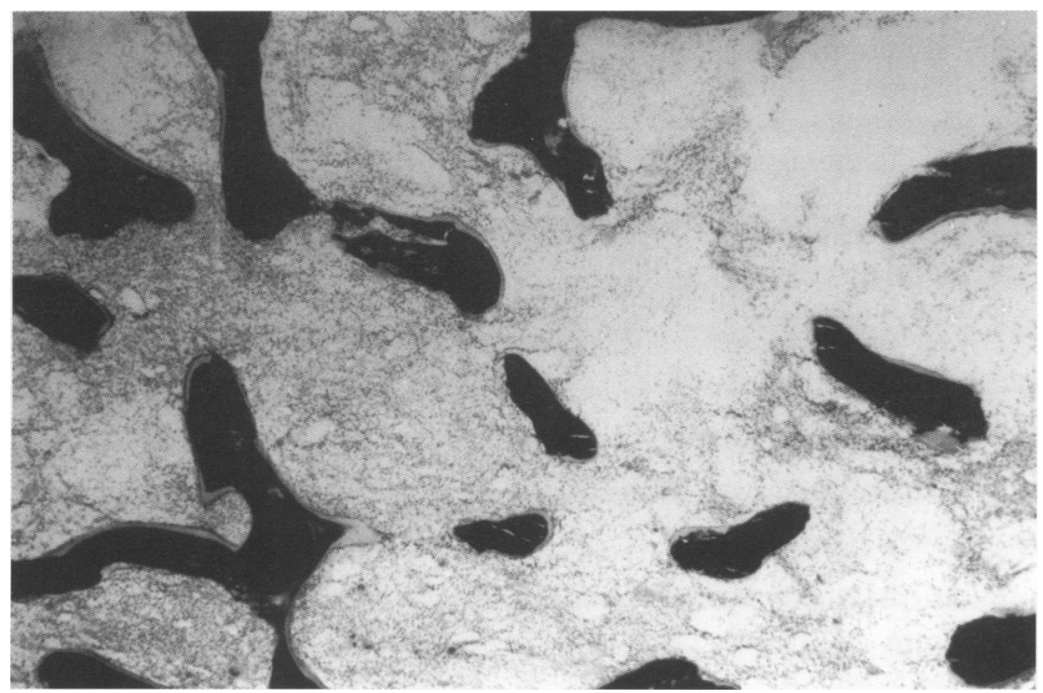

Figure 2 Right iliac crest biopsy specimen. Osteoid demonstrating normal mineralisation. (Toluidine blue/EDTA; original magnification $\times 50$.) presentation and clinical courses are likely to reflect variable expression of the alkaline phosphatase gene defect.

Treatment of hypercalcaemia by dietary restriction of calcium in infantile hypophosphatasia is reported to be variably successful. ${ }^{2}$ Other methods have been used alone or in combination, including steroids, ${ }^{3}$ dietary vitamin D restriction, reduced exposure to sunlight, ${ }^{4}$ and oral phosphate supplementation. ${ }^{3}$ Although these methods have been successful initially, rebound hypercalcaemia is well documented. ${ }^{3}$

Dental abnormalities in hypophosphatasia result principally from premature loss of the primary dentition, with histological examination demonstrating absent predentine mineralisation, absent cementum and variable reduction in dentine. Secondary dentition is reported to develop normally in some patients; however, enamel hypoplasia and caries are seen in others with radiological evidence of enlarged pulp chambers and root canals. ${ }^{2}$

The skeletal histology seen in perinatal and infantile hypophosphatasia is similar, ${ }^{2}$ with the more severe changes seen in the lethal perinatal form than in those survivors of infantile hypophosphatasia, although accurate interpretation is hampered by the lack of normal controls in this age group.

In the infantile form changes are reported in osseous regions producing metaphyseal and diaphyseal abnormalities with trabecular widening, distortion, foci of uncalcified cartilage, and increased osteoid secondary to reduced mineralisation. ${ }^{2}$ The cartilaginous growth plate is also affected, particularly the hypertrophic zone which may be notably widened with distortion of the columnar arrangement of the chondrocytes. $^{2}$ The calcified zones also are severely affected as a result of demineralisation to such an extent that in some cases this zone is absent. $^{2}$

Some reports of infantile hypophosphatasia have shown osteoblasts of normal number and morphology. Other reports ${ }^{25}$ have demonstrated variability of osteoclastic numbers and activity, with both abnormal remodelling and active resorption being observed in different studies.

Remineralisation of bone in infantile hypophosphatasia has been reported to occur both spontaneously and following treatment with alkaline phosphatase rich serum. ${ }^{6}$ In the latter cases remineralisation has been associated with an increase in serum alkaline phosphatase activity.

The serial clinical, biochemical, radiological and, in particular, histological improvement despite persistently low alkaline phosphatase activity seen in the present patient is consistent with previous reports, ${ }^{2}$ but is more difficult to explain. Some authors have suggested such spontaneous improvement may reflect, in part, an influence of transplacental maternal alkaline phosphatase, but we feel the histological data in the present case suggests a progressive increase in the bioactivity of tissue alkaline phosphatase, as has been demonstrated in experimental models. ${ }^{7}$ 
1 Brenton DP, Krywawych S. Hypophosphatasia. Clin Rheum Dis 1986;12:771-89.

2 Caswell AM, Whyte MP, Russell RGG. Hypophosphatasia and the extracellular metabolism of inorganic pyrophosphate: Clinical and laboratory aspects. Crit Rev Clin Lab Sci 1991;28:175-223.

3 Wolfish NM, Heick H. Hyperparathyroidism and infantile hypophosphatasia: effect of prednisone and vitamin $\mathrm{K}$ therapy. $\mathcal{F}$ Pediatr 1979;95:1079.

4 Opshaug O, Maurseth K, Howlid H, Aksnes L, Aarskog D. Vitamin D metabolism in hypophosphatasia. Acta Pediatr Scand 1982;71:517.
5 Goldfischer S, Johnson AB, Morecki R. Hypophosphatasia. A cytochemical study of phosphatase activities. Lab Invest 1976;43:106

6 Whyte MP, McAllister WH, Patton LS, Magill HL, Fallon MD, Lorentz WB, et al. Enzyme replacement therapy for fantile hypoline sions of alkaline phosphatase rich Paget plasma: results in
three additional patients. $\mathcal{F}$ Pediatr 1984;105:926.

7 Jung A, Russell RGG, Bisaz S, Morgan DB, Fleisch H. Fate of intravenously injected pyrophosphate $32-\mathrm{P}$ in dogs. $\mathrm{Am}$ f Physiol 1970;218: 1757 .

\title{
Does storage of sputum specimens adversely affect culture results?
}

\author{
F K Gould, R Freeman, S Hudson, J Magee, D Nelson, R Stafford, P R Sisson
}

\begin{abstract}
It has been recommended that samples submitted for microbiological examination should be retained for 48 hours after issue of the final report. In order to ascertain whether reproducible results could be achieved following storage of sputum specimens, two laboratories each re-cultured 100 samples 48 hours after their report had been issued and a further laboratory re-cultured 100 samples 48 hours after receipt. Discordant results were obtained in only $5-25 \%$ of specimens, indicating that potential respiratory pathogens could survive storage. ( Clin Pathol 1996;49:684-686)
\end{abstract}

Keywords: sputum samples storage, respiratory pathogens, microbiological examination.

\section{Department of Microbiology, Freeman Hospital, Newcastle upon Tyne F K Gould D Nelson}

\section{Newcastle Public} Health Laboratory, General Hospital, Newcastle upon Tyne R Freeman

J Magee

P R Sisson

\section{Department of} Microbiology, Queen Elizabeth Hospital, Gateshead, Tyne and

Wear

$S$ Hudson

R Stafford

Correspondence to Dr F K Gould

Microbiology Department, Freeman Hospital, High Heaton,

Hewcastle upon Tyne NE7

Newcast

Accepted for publication 16 April 1996
It has been a widely held belief amongst microbiologists that potential pathogens such as Streptococcus pneumoniae and Haemophilus influenzae survive poorly in clinical specimens and so they advocate rapid processing of samples such as sputum and pleural fluid in order to maximise their diagnostic potential. ${ }^{1-3}$ It was therefore surprising that the Working Party on the Retention and Storage of Pathological Records and Archives recommended that all specimens should be retained for a period of 48 hours following issue of a final report, ${ }^{4}$ presumably to permit confirmation of the original culture results. In order to ascertain whether reproducible results could be obtained from stored sputum samples a prospective study was coordinated in three laboratories using their individual standard operating procedures (SOPs).

\section{Methods}

One hundred consecutive sputum specimens received for routine culture and sensitivity in two diagnostic microbiology laboratories (A and $\mathrm{B}$ ) were retained at $4^{\circ} \mathrm{C}$ for at least two working days following the issue of the final $\rightarrow$ report. A third laboratory (C) retained 100 sputum specimens as above for 48 hours following receipt. All the samples were then $\stackrel{5}{\rightarrow}$ re-cultured, usually by the same individual, but $\overrightarrow{0}$ without knowledge of the previous result, according to each laboratory's SOP and the $\square$ results compared. Invasive samples such as those obtained from bronchoscopy were excluded, as were specimens from patients known to be bronchiectatic in which overgrowth with Pseudomonas sp could be expected.

The SOP for the investigation of routine sputum samples at each laboratory was briefly as follows:

\section{LABORATORY A}

Samples were refrigerated on receipt at the laboratory and processed the same day. Samples were vortexed with equal volumes of ster- $\frac{\text { O }}{3}$ ile deionised water until completely homoge- $>$ neous and then plated onto half $5 \%$ horse blood/half heated blood agar and incubated $N$ overnight at $35^{\circ} \mathrm{C}$ in $5 \% \mathrm{CO}_{2}$. Plates were then returned to the incubator and re-examined after a further 24 hours' incubation.

\section{LABORATORY B}

Samples were processed on the day of receipt. $\stackrel{\oplus}{\rightarrow}$ Each specimen was homogenised using dithiothreitol (Sputasol, Unipath Ltd) before being 0 cultured onto $5 \%$ horse blood, heated blood $\mathbb{D}$

Table 1 Comparison of results of sampling according to routine practice with sampling two to three days after the clinical report had been issued (laboratories $A$ and B) or 48 hours after receipt (laboratory $C$ )

\begin{tabular}{lccc}
\hline & \multicolumn{3}{c}{ Laboratory } \\
\cline { 2 - 4 } & $A$ & $B$ & $C$ \\
\hline No of specimens yielding concordant results & 79 & 77 & 95 \\
$\quad$ Significant pathogen(s) & $(21)$ & $(25)$ & $(29)$ \\
No significant pathogen(s) & $(58)$ & $(52)$ & $(66)$ \\
No of specimens yielding discordant results & 21 & 23 & 5 \\
Total & 100 & 100 & 100
\end{tabular}

